\title{
Assimilation of Earth rotation parameters into a global ocean model: excitation of polar motion
}

\author{
J. Saynisch ${ }^{1}$, M. Wenzel ${ }^{2}$, and J. Schröter ${ }^{2}$ \\ ${ }^{1}$ Helmholtz Centre Potsdam, GFZ German Research Centre for Geosciences, Section 1.3: Earth System Modelling, \\ Potsdam, Germany \\ ${ }^{2}$ Alfred Wegener Institute for Polar and Marine Research, Climate Sciences - Ocean Dynamics, Bremerhaven, Germany \\ Received: 26 May 2011 - Revised: 19 August 2011 - Accepted: 24 August 2011 - Published: 8 September 2011
}

\begin{abstract}
The oceanic contribution to Earth rotation anomalies can be manifold. Possible causes are a change of total ocean mass, changes in current speed or location and changes in mass distribution. To derive the governing physical mechanisms of oceanic Earth rotation excitation we assimilate Earth rotation observations with a global circulation ocean model. Before assimilation, observations of length of day and polar motion were transformed into estimates of ocean angular momentum. By using the adjoint 4D-VAR assimilation method we were able to reproduce these estimated time series. Although length of day was assimilated simultaneously the analysis in this paper focuses on the oceanic polar motion generation. Our results show that changes in mass distribution and currents contribute to oceanic polar motion generation. Both contributions are highly correlated and show similar amplitudes. The changes in the model done by the assimilation procedure could be related to changes in the atmospheric forcing. Since for geometrical reasons the change of total ocean mass does not project on polar motion, we conclude that the polar motion is mainly generated by a geostrophic response to atmospheric momentum forcing. In geostrophic currents mass displacement and current speed entail each other. This way the large similarity of mass and current generated ocean angular momentum can be explained.
\end{abstract}

\section{Introduction}

The theory of internal Earth rotation excitations is based on a closed system with no external torques. Under such conditions the Earth's angular momentum is conserved. This is stated in the Euler-Liouville equation. Applying a variational

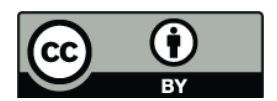

Correspondence to: J. Saynisch (saynisch@gfz-potsdam.de) ansatz to the Euler-Liouville equation gives the following partial differential equation:

$$
\begin{aligned}
\frac{\dot{m_{1}}}{\sigma_{\mathrm{ch}}}+m_{2} & =\frac{\dot{\chi_{1}}}{\Omega}+\chi_{2} \\
-\frac{\dot{m_{2}}}{\sigma_{\mathrm{ch}}}+m_{1} & =-\frac{\dot{\chi_{2}}}{\Omega}+\chi_{1} \\
\dot{m_{3}} & =-\dot{\chi_{3}}
\end{aligned}
$$

Here, the $m_{i}$ are small perturbations of Earth's mean angular velocity $\boldsymbol{\Omega}=\Omega(0,0,1)$, i.e. $\tilde{\boldsymbol{\Omega}}=\Omega\left(m_{1}, m_{2}, 1+m_{3}\right)$. These perturbations are excited by the forcing functions $\chi_{i}$. The $m_{1}$ and $m_{2}$ are labeled polar motion (PM). Excited only once they would describe an oscillation of frequency $\sigma_{\mathrm{ch}}$. This free nutation of the Earth is called the Chandler-Wobble (Lambeck, 1980). Since the Chandler-Wobble is a damped phenomenom $\sigma_{\mathrm{ch}}$ is a complex number. Note that due to sustained and variable forcing (via $\chi_{1}$ and $\chi_{2}$ ) the PM-signal contains various frequencies different from $\sigma_{\mathrm{ch}}$.

The changes of the mean absolute value of Earth's angular velocity $\Omega$ are described by the so called length of day (LOD) changes, i.e. $m_{3}$. The results of the presented study as far as LOD is concerned are described in Saynisch et al. (2011). In the following we focus on the implications for PM only.

We follow the formulation of Barnes et al. (1983) where the forcing functions contain angular momentum anomalies only. Furthermore, secondary effects of rotational deformation and loading are considered:

$$
\begin{aligned}
& \chi_{1} \equiv \frac{1}{\Omega(C-A)}\left(\Omega \Delta \mathbb{J}_{31}+1.43 \Delta L_{\mathrm{r} 1}\right) \\
& \chi_{2} \equiv \frac{1}{\Omega(C-A)}\left(\Omega \Delta \mathbb{J}_{32}+1.43 \Delta L_{\mathrm{r} 2}\right)
\end{aligned}
$$

In this formulation the $\chi_{i}$ are called effective angular momentum functions (Barnes et al., 1983). Here, $A$ and $C$ are the principal moments of inertia of the solid Earth. Based on Eqs. (4) and (5), changes in the $\chi_{i}$ and therefore changes in the Earth's rotation can be caused by a changing tensor

Published by Copernicus Publications on behalf of the European Geosciences Union and the American Geophysical Union. 
of inertia $\mathbb{J}$ or a change of angular momentum relative to the Earth's surface $L_{\mathrm{r}}$ or both. Accordingly, we call the two terms of the Eqs. (4) and (5) mass-term respective relativeterm.

The paper is structured as follows. We briefly describe the utilized models and data sets in Sect. 2. In Sect. 3, we present the results of the data assimilation-simulation in comparison to a reference-simulation. We summarize and conclude in Sect. 4. A mathematical introduction to the adjoint assimilation method can be found in Appendix A.

\section{Methodology}

This study uses exactly the same experimental setting as Saynisch et al. (2011). A detailed description and discussion of utilized methodology, data sets and models can be found therein. An overview will be given here.

We use the global circulation ocean model of MaierReimer et al. (1993). In our configuration the model has a free surface and is mass conserving. The resolution is $2.1^{\circ}$ and the time step is 10 days. It is forced with momentum, heat and freshwater flux. The forcing is a recomposition of empirical orthogonal functions obtained from atmospheric reanalyses. The model's angular momentum functions are calculated as in Eqs. (4) and (5) and compared with the observations in a weighted quadratic cost function. The weighting was derived from the misfit of atmospheric angular momentum functions from ECMWF and NCEP products.

The observations are based on daily C04 Earth rotation parameters (PM and LOD) provided by the IERS (Gambis, 2004; Vondrak and Richter, 2004). The measurements are provided in a reference system called celestial intermediate pole (CIP, McCarthy and Petit, 2004). The conversion of this time series into angular momentum function follows Gross (1992). Subsequently, we subtract angular momentum functions calculated from models of the atmosphere (ECMWF, ERA-40, Uppala et al., 2005) and land hydrology (HDM, Hagemann and Dümenil, 1998). Details about influence and quality of the HDM contributions can also be found in Saynisch et al. (2011). A 1.5-yr highpass-filter removes low-frequency anomalies that are supposed to originate in the Earth's core and mantle (Pais and Hulot, 2000). The residual is supposed to represent ocean angular momentum (OAM) to a large extend.

To ensure a realistic ocean trajectory additional data sets were assimilated simultaneously. These include climatological salt contents, temperatures and velocities (Gouretski and Koltermann, 2004; Chapman, 1998; Conkright et al., 2002) as well as respective time series for the upper ocean (Willis et al., 2004) and the sea surface elevation (Reynolds et al., 2002).

An adjoint 4D-VAR assimilation procedure was used (see, e.g. Le Dimet and Talagrand, 1986). This method has the advantage of conserving the ocean model's physics. The
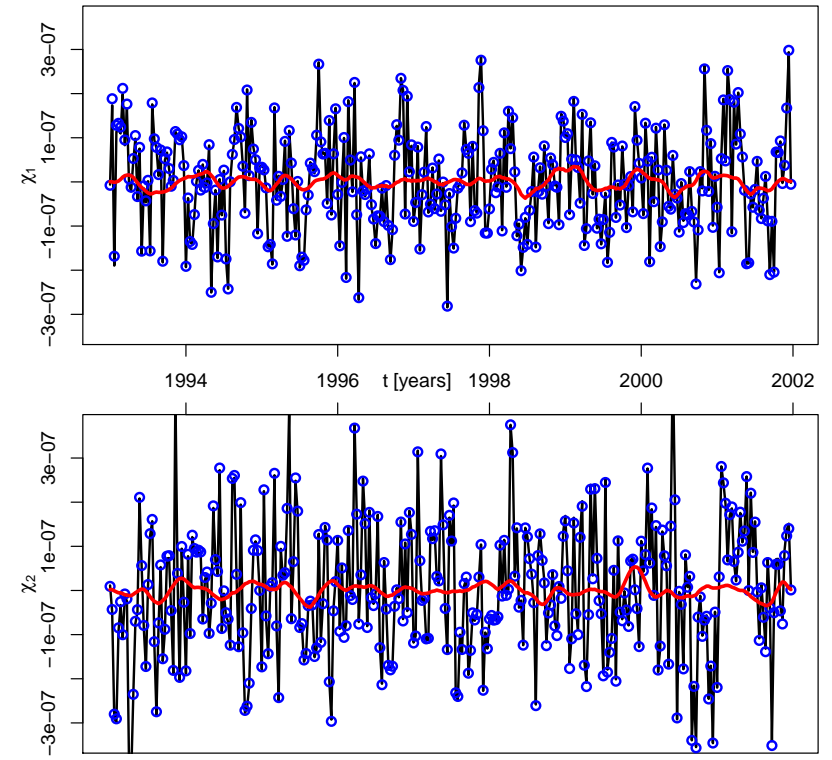

Fig. 1. Equatorial oceanic angular momentum functions. Reference-simulation (red line). Assimilation-simulation (blue dots). OAM observations (black line).

assimilated time span was chosen to cover the beginning of 1993 till the end of 2001. The control vector, i.e. the model values that are changeable by the assimilation procedure before the ocean model is rerun, were the initial ocean state (i.e. velocities, temperatures, salinities and sea surface elevations) and the surface fluxes of every time step (i.e. heat, freshwater and momentum). Mathematical details about the adjoint method can be found in Appendix A.

In addition to the assimilation-simulation a referencesimulation was created which assimilated no OAM observations but the above mentioned oceanographic data sets only. This simulation is used for the evaluation of influence and success of the OAM assimilation procedure.

\section{Results}

As mentioned, this paper focuses on PM only. Results concerning LOD can be found in Saynisch et al. (2011). The fit of modeled OAM (before and after assimilation) with the observation based OAM-estimates is shown in Fig. 1.

It is evident that the variance of the reference-simulation is too small to reproduce the OAM-observations. In contrast, the ocean model after the assimilation is able to reproduce the observations well. The rms of the $\chi_{1}$-misfit reduces by two orders of magnitude from the reference-simulation's $1.1 \times 10^{-07}$ to $3.8 \times 10^{-09}$ after assimilation. In case of $\chi_{2}$ the rms-misfit reduces comparably from $1.5 \times 10^{-07}$ to $5.4 \times 10^{-09}$. The rms-reduction is not only the result of higher ocean model's OAM-variance, i.e. amplitude, but also 

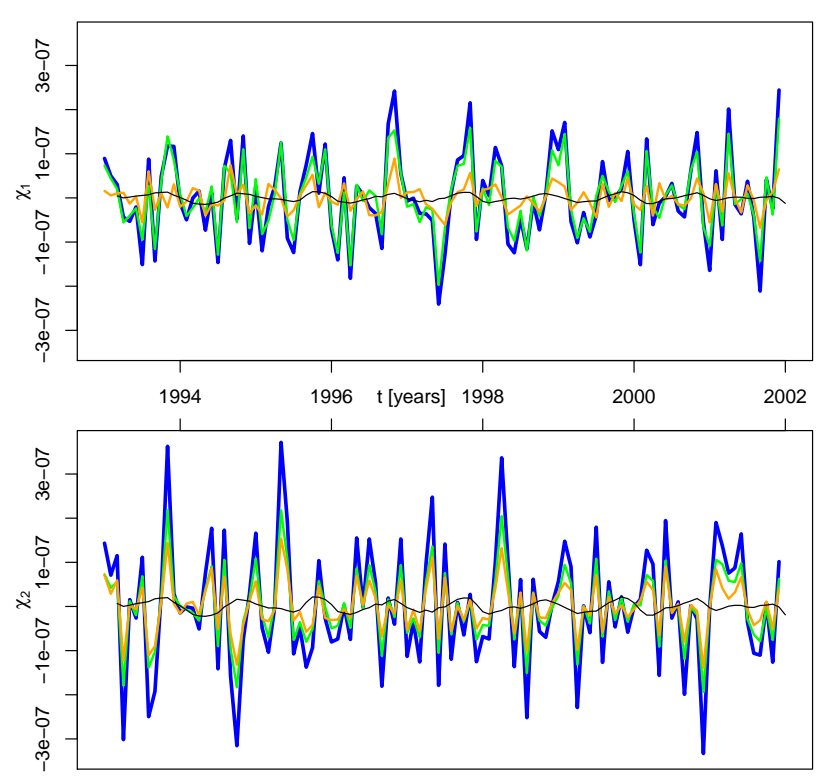

Fig. 2. Ocean angular momentum of the assimilation-simulation. Total ocean angular momentum (blue). Relative-term (orange). Mass-term (green). Part of the mass-term which is due to total ocean mass change (black).

results from an expansion of the model's temporal variability to shorter time-scales.

Subsequently, we want to identify the mechanisms which are responsible for this change in modeled OAM amplitude and temporal variability. To this end we divided the modeled OAM into contributions due to the mass-term and due to the relative-term (see Eqs. 4 and 5).

The respective contributions are plotted in Fig. 2. Here it becomes evident that the good fit of the assimilationsimulation can be likewise attributed to changes in mass-term and relative-term. Both share the high amplitudes and the fast temporal variability of Fig. 1 (blue dots). Interestingly, massterm and relative-term are highly correlated. Furthermore, to clarify whether the mass-term is due to redistribution of mass or due to total ocean mass change we calculated these contributions as well. The latter is explicitely plotted in Fig. 2 (black line). It can be stated that the contributions to oceanic PM from total ocean mass change are small. This is not very surprising since the functions which govern the transformation of a global mass distribution into $\mathbb{J}_{31}$ and $\mathbb{J}_{32}$ are spherical harmonics of degree 2 and order 1 . These functions sum up to zero in a global integration. This corresponds to the fact that if the Earth would have a globally uniform mass distribution there would be no PM, i.e. no Chandler-Wobble. The same would apply for a global ocean in the absence of continents. Since even local changes in ocean mass lead very fast to a globally uniform change in bottom pressure, the contribution to PM would vanish in this case. Therefore, it is

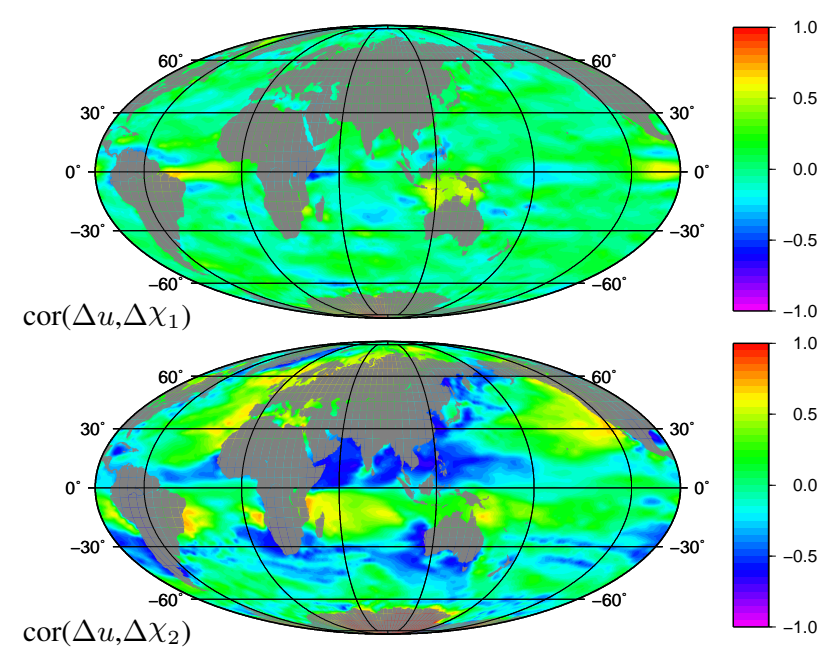

Fig. 3. Temporal correlation of the changes induced by the assimilation procedure: correlations between changes in the model's zonal surface velocity and the change in modeled $\chi_{1}$ (top), respectively $\chi_{2}$ (bottom).

only due to the symmetry-breaking of the continents that the black line in Fig. 2 is not zero. Consequently, the major fraction of the mass-term can be attributed to the redistribution of oceanic mass.

In search for the physical mechanisms that are responsible for the generation of the modeled PM-excitation we focused on the changes in the model-state done by the assimilation. Therefore, we calculated the differences between the model-states of the assimilation-simulation and the reference-simulation. These differences were correlated with the respective differences of the modeled OAM-functions. We found strong correlations between the surface velocities and $\chi_{1}$ and $\chi_{2}$ (see Figs. 4 and 3). Especially, high correlations can be found between zonal velocity and $\chi_{2}$. The same amounts for the meridional velocity and $\chi_{1}$. These correlations vanish quickly with depth and show the typical bandstructure of the global atmospheric circulation.

\section{Summary and conclusion}

To derive the governing physical mechanisms of oceanic polar motion excitation we assimilated Earth rotation observations, namely polar motion and length of day, with a global circulation ocean model. We subtracted non-oceanic contributions from the observations and used an adjoint method for the assimilation of the residuals. The correlation of modeled and observed oceanic angular momentum was enhanced substantially by the assimilation. Analysis of the ocean model's state after assimilation and the comparison with a referencesimulation brought the following results: total ocean mass change has a negligible contribution to polar motion. Contributions from anomalies in mass distribution and currents 


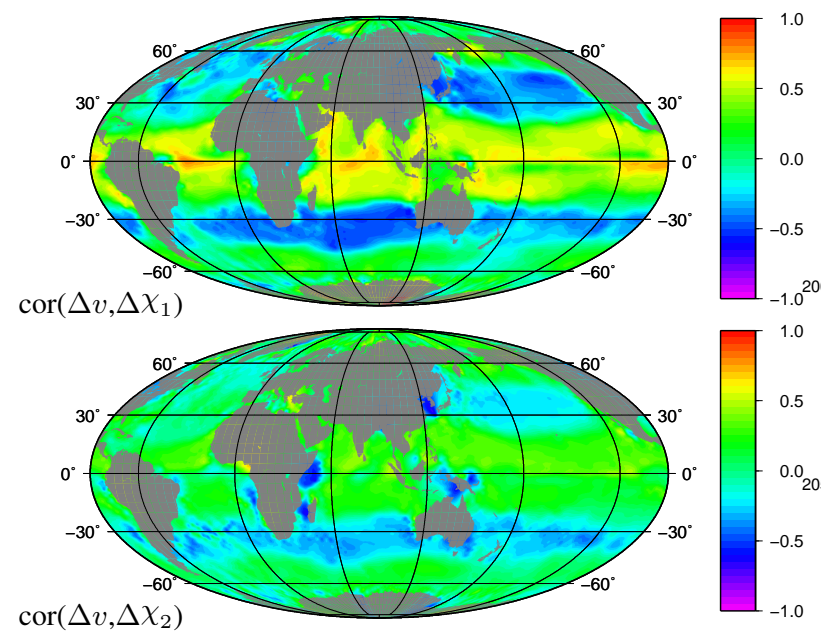

Fig. 4. Temporal correlations of the changes induced by the assimilation procedure: correlations between changes in the model's meridional surface velocity and the change in modeled $\chi_{1}$ (top), respectively $\chi_{2}$ (bottom).

are very similar in size. Additionally, both contributions are highly correlated. The assimilation procedure changed the atmospheric momentum flux into the ocean to reduce the misfit between model and observation. For the governing physical processes we conclude the following: wind-induced Ekman-transports lead to changes in the relative-term of the OAM. As a consequence of the displaced water changes in the ocean pressure-fields occur. This, in turn, initiates geostrophic currents. Since geostrophic streamlines coincide with sea level contours the relative-term and the mass-term get further entangled. In this way the high correlations between mass-term and relative-term can be explained. Since these currents are limited to the upper ocean, i.e. the Ekmanlayer, the vanishing of the mentioned correlations in deeper layers can be explained.

\section{Appendix A}

Here, we present the mathematics behind the adjoint assimilation technique. More details can be found in Le Dimet and Talagrand (1986).

Given a model $\mathbb{M}$ that operates on a set of parameters $\mathbf{P}$ we can extract a desired observable $\mathbf{X}$ by applying an observation operator $\mathbb{H}$ to the model:

$\mathbf{X}=\mathbb{H} \mathbb{M} \mathbf{P}$

Consequently $\mathbf{X}$ depends on $\mathbf{P}$ as follows, where the $\delta$ denote small deviations:

$$
\delta \mathbf{X}=\underbrace{\left.\frac{\partial \mathbb{H}}{\partial \mathbb{M}} \frac{\partial \mathbb{M}}{\partial \mathbf{P}}\right|_{\mathbf{P}_{0}} \delta \mathbf{P}}_{\mathbb{T}}
$$

If we linearize the differential operator $\mathbb{T}$ it is called tangent linear model. Note that this relation is not constant but depends on a certain set of parameters $\mathbf{P}_{0}$. Normally, in the field of data assimilation the $\delta \mathbf{X}$ is given, e.g. as the misfit between modeled observable $\mathbf{X}_{\mathrm{M}}$ and its observation $\mathbf{X}_{\mathrm{O}}$. That misfit is usually stated as a quadratic cost function, here written as a scalar-product:

$$
J\left(\mathbf{X}_{\mathrm{M}}\right)=<\mathbf{X}_{\mathrm{M}}-\mathbf{X}_{\mathrm{O}}, \mathbf{X}_{\mathrm{M}}-\mathbf{X}_{\mathrm{O}}>
$$

Now, a $\delta \mathbf{P}$ is searched that produces the desired change in model output $\delta \mathbf{X}=\mathbf{X}_{\mathrm{O}}-\mathbf{X}_{\mathrm{M}}$. In other words we want to invert relation (A2). To this end, we make a variational ansatz, where $\nabla_{\mathbf{X}}$ denotes the gradient in observation space:

$$
J=J\left(\mathbf{X}_{\mathbf{0}}\right)+<\left.\nabla_{\mathbf{X}} J^{T}\right|_{\mathbf{x}_{\mathbf{0}}}, \delta \mathbf{X}>+O\left(\delta \mathbf{X}^{2}\right)
$$

respectively:

$$
=J\left(\mathbf{P}_{\mathbf{0}}\right)+<\left.\nabla_{\mathbf{P}} J^{T}\right|_{\mathbf{P}_{\mathbf{0}}}, \delta \mathbf{P}>+O\left(\delta \mathbf{P}^{2}\right)
$$

Consequently, in the latter equation $\nabla_{\mathbf{P}}$ represents the gradient with respect to the parameter space. Inserting Eq. (A2) in Eq. (A4) gives:

$$
\begin{aligned}
\delta J & =<\left.\nabla_{\mathbf{X}} J^{T}\right|_{\mathbf{X}_{\mathbf{0}}}, \mathbb{T} \delta \mathbf{P}> \\
& =<\left.\mathbb{T}^{*} \nabla_{\mathbf{X}} J^{T}\right|_{\mathbf{x}_{\mathbf{0}}}, \delta \mathbf{P}>
\end{aligned}
$$

Here $\mathbb{T}^{*}$ denotes the adjoint of $\mathbb{T}$, hence the name of the method. Now, comparing (A7) with (A5) gives the following identity:

$$
\begin{aligned}
\delta J & =<\left.\nabla_{\mathbf{P}} J^{T}\right|_{\mathbf{P}_{\mathbf{0}}}, \delta \mathbf{P}> \\
& =<\left.\mathbb{T}^{*} \nabla_{\mathbf{X}} J^{T}\right|_{\mathbf{X}_{\mathbf{0}}}, \delta \mathbf{P}>
\end{aligned}
$$

By comparing the left entries of the scalar-product we get:

$$
\left.\nabla_{\mathbf{P}} J^{T}\right|_{\mathbf{P}_{\mathbf{0}}}=\left.\mathbb{T}^{*} \nabla_{\mathbf{X}} J^{T}\right|_{\mathbf{X}_{\mathbf{0}}}
$$

Therefore, in the case of a quadratic cost function the gradient of $J$ with respect to the parameter space is simply given by the adjoint tangent linear model acting on the misfit of model and observation:

$$
\left.\nabla_{\mathbf{P}} J^{T}\right|_{\mathbf{P}_{\mathbf{0}}}=2 \mathbb{T}^{*}\left(\mathbf{X}_{\mathrm{M}}-\mathbf{X}_{\mathrm{O}}\right)
$$

Knowing this identity we can now minimize $J$ iteratively, where $\epsilon$ is a small number governing the step-size:

$$
\begin{aligned}
\mathbf{P}_{i+1} & =\mathbf{P}_{i}+\epsilon \mathbb{T}_{i}^{*}\left(\mathbf{X}_{\mathrm{M}_{\mathrm{i}}}-\mathbf{X}_{\mathrm{O}}\right) \\
\mathbf{X}_{\mathrm{M}_{\mathrm{i}+1}} & =\mathbb{H} \mathbb{M} \mathbf{P}_{i+1} \\
\mathbb{T}_{i+1} & =\left.\frac{\partial \mathbf{X}}{\partial \mathbf{P}}\right|_{\mathbf{P}_{i+1}}
\end{aligned}
$$


Several passes of $\mathbb{M}$ and $\mathbb{T}$, i.e. the forward and the backward model, are required before $\mathbf{X}_{\mathrm{M}}$ converges to $\mathbf{X}_{\mathrm{O}}$. The important fact is that $\mathbb{M}$, i.e. the incorporated physics, stays intact during assimilation and only $\mathbf{P}$ gets changed to produce the desired output.

Acknowledgements. The project is part of the research unit "Earth Rotation and Global Dynamic Processes" which is funded by the "German Research Foundation". This work could not have be done without the supply of ERA-40 data from the European Centre for Medium-Range Weather Forecasts, Earth rotation data from the International Earth rotation and Reference systems Service and facilities from the Alfred Wegener Institute for Polar and Marine Research and the German High Performance Computing Centre for Climate- and Earth System Research. We thank the following colleagues for nourishing discussions and substantial remarks: Dirk Olbers, Martin Losch and Sergey Danilov. The same accounts for the editor Reik Donner and the reviewers of this manuscript.

Edited by: R. Donner

Reviewed by: T. Nilsson and two other anonymous referees

\section{References}

Barnes, R. T. H., Hide, R., White, A. A., and Wilson, C. A.: Atmospheric angular-momentum fluctuations, Length-Of-Day changes and Polar Motion, Proc. R. Soc. London Ser. A-Math. Phys. Eng. Sci., 387, 31-73, 1983.

Chapman, P.: The world ocean circulation experiment (WOCE), Mar. Technol. Soc. J., 32, 23-36, 1998.

Conkright, M. E., Locarnini, R. A., Garcia, H. E., O’Brien, T. D.and Boyer, T. P., Stephens, C., and Antonov, J. I.: World Ocean Atlas 2001: Objective analysis, data statistics and figures, Tech. rep., National Oceanographic Data Center, CD-ROM Dokumentation, 2002.

Gambis, D.: Monitoring Earth orientation using space-geodetic, techniques: state-of-the-art and prospective, J. Geodesy, 78, 295-303, 2004.

Gouretski, V. V. and Koltermann, K. P.: WOCE global hydrographic climatology, Tech. Rep. 35, Bundesamt für Seeschifffahrt und Hydrographie, 2004.

Gross, R. S.: Correspondence between theory and observations of polar motion, Geophys. J. Int., 109, 162-170, 1992.
Hagemann, S. and Dümenil, L.: Documentation for the Hydrological Discharge Model, Tech. Rep. 17, Deutsches Klimarechenzentrum (DKRZ), Hamburg, Germany, 1998.

Lambeck, K.: The Earth's variable rotation, Cambridge University Press, 1980.

Le Dimet, F. and Talagrand, O.: Variational algorithms for analysis and assimilation of meteorological observations - theoretical aspects, Tellus Ser. A-Dyn. Meteorol. Oceanol., 38, 97-110, 1986.

Maier-Reimer, E., Mikolajewicz, U., and Hasselmann, K.: Mean circulation of the Hamburg LSG OGCM and its sensitivity to the thermohaline surface forcing, J. Phys. Oceanogr., 23, 731-757, 1993.

McCarthy, D. D. and Petit, G.: IERS Conventions (2003), Tech. Rep. 32, IERS, 2004.

Pais, A. and Hulot, G.: Length of day decade variations, torsional oscillations and inner core superrotation: evidence from recovered core surface zonal flows, Phys. Earth Planet. Inter., 118, 291-316, 2000.

Reynolds, R. W., Rayner, N. A., Smith, T. M., Stokes, D. C., and Wang, W. Q.: An improved in situ and satellite SST analysis for climate, J. Climate, 15, 1609-1625, 2002.

Saynisch, J., Wenzel, M., and Schröter, J.: Assimilation of Earth rotation parameters into a global ocean model: length of day excitation, J. Geodesy, 85, 67-73, 2011.

Uppala, S. M., Kallberg, P. W., Simmons, A. J., Andrae, U., Bechtold, V. D., Fiorino, M., Gibson, J. K., Haseler, J., Hernandez, A., Kelly, G. A., Li, X., Onogi, K., Saarinen, S., Sokka, N., Allan, R. P., Andersson, E., Arpe, K., Balmaseda, M. A., Beljaars, A. C. M., Van De Berg, L., Bidlot, J., Bormann, N., Caires, S., Chevallier, F., Dethof, A., Dragosavac, M., Fisher, M., Fuentes, M., Hagemann, S., Holm, E., Hoskins, B. J., Isaksen, L., Janssen, P. A. E. M., Jenne, R., McNally, A. P., Mahfouf, J. F., Morcrette, J. J., Rayner, N. A., Saunders, R. W., Simon, P., Sterl, A., Trenberth, K. E., Untch, A., Vasiljevic, D., Viterbo, P., and Woollen, J.: The ERA-40 re-analysis, Q. J. R. Meteorol. Soc., 131, 29613012, 2005.

Vondrak, J. and Richter, B.: International Earth Rotation and Reference Systems Service (IERS), J. Geodesy, 77, 585-678, 2004.

Willis, J. K., Roemmich, D., and Cornuelle, B.: Interannual variability in upper ocean heat content, temperature, and thermosteric expansion on global scales, J. Geophys. Res.-Oceans, 109, C12036, doi:10.1029/2003JC002260, 2004. 